\title{
A METHOD OF DESIGNING MAGNETIC CHANNELS OF IMPROVED FIELD QUALITY FOR SUPERCONDUCTING CYCLOTRONS
}

\author{
P. R. Sarma, V. S. Pandit and R. K. Bhandari \\ Variable Energy Cyclotron Centre, Bidhannagar, Kolkata-700 064, India
}

\begin{abstract}
Magnetic channels are used in the extraction system of superconducting cyclotrons for focusing and extracting the high energy beam. In order to preserve the beam quality, the field gradient produced by the channels should be constant. We have evolved a procedure based on the linear programming technique for optimizing the geometry of magnetic channels. With this, we have reduced the deviation in the field gradient not only in the median plane but through the beam aperture.
\end{abstract}

\section{INTRODUCTION}

In high energy cyclotrons, especially in superconducting cyclotrons, in addition to electrostatic deflectors, passive magnetic channels are used for extracting the beam. These channels use iron bars to lower the magnetic field in the extraction region thereby helping the extraction process. These also generate a positive field gradient (opposite to radially defocusing fringe field gradient) and so provide a radial focusing force. At Variable Energy Cyclotron Centre in Calcutta, a K500 superconducting cyclotron, similar to the one at Michigan State University[1], is under construction. The extraction system uses 9 magnetic channels[2].

Typical magnetic channels consist of three iron bars, one placed at a lower radius and two other at a higher radius with respect to a beam orbit (Fig. 1)[3]. In the strong field of the superconducting cyclotron these bars become magnetically saturated. The single bar on the left reduces the field near it and the other two bars on the right increase the field at higher radii. The combined effect is a negative field in the centre of the channel and a radially focusing gradient within the aperture occupied by the beam. Such magnetic channels have been used in the superconducting cyclotrons at Chalk-River[4], NSCL at MSU[5] and at Milan[6].

In order to preserve the beam quality, it is necessary that the field gradient of the channel be constant along the radial direction not only in the median plane but also in the planes above and below it, because the beam has finite radial and vertical dimensions. The field gradient is determined by the dimensional parameters of the channel geometry. Recently we developed a method[7], based on the linear programming technique, to optimize the channel parameters for reducing the deviations of the field gradient. We considered only purely rectangular bar shapes there. Here we have improved and used the method to further improve the field quality of the channel by considering other shapes.

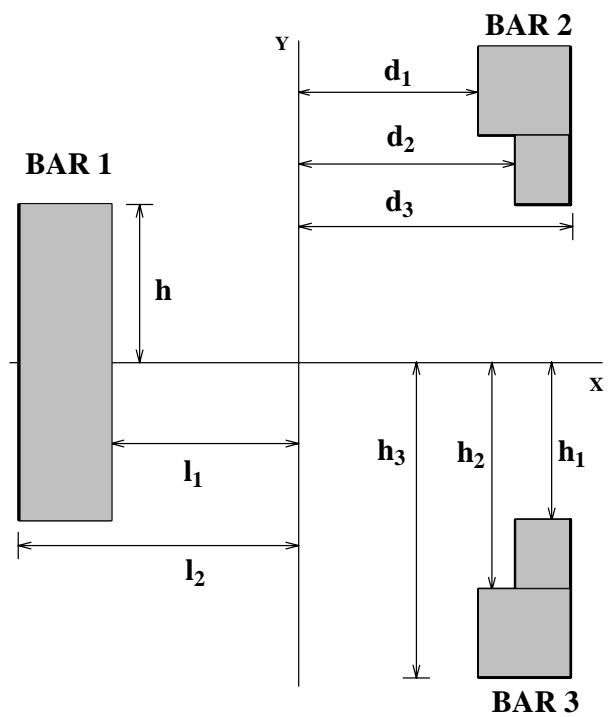

Figure 1: Channel geometry showing various dimensional parameters.

\section{CALCULATION OF MAGNETIC FIELD}

Since the iron bars are completely saturated, the field due to the bars is the same as that due to current sheets on the vertical surfaces of the bars. Thus $B_{z}(x, y)$, the vertical component of the field at a point $(x, y)$ due to a bar of thickness $t$ and height $2 h$, placed at a distance $d$ from the origin is

$$
\begin{aligned}
B_{z}(x, y)= & A\left[\int_{-h-y}^{h-y} \frac{(d-x)}{(d-x)^{2}+y^{2}} d y-\right. \\
& \left.\int_{-h-y}^{h-y} \frac{(d+t-x)}{(d+t-x)^{2}+y^{2}} d y\right]
\end{aligned}
$$

where $A=-3.375 \mathrm{kG}$ in the case of iron saturated to 21.4 $\mathrm{kG}$. This can be simplified to

$$
\begin{aligned}
B_{z}(x, y)= & A\left[\tan ^{-1} \frac{h-y}{d-x}-\tan ^{-1} \frac{h-y}{d+t-x}+\right. \\
& \left.\tan ^{-1} \frac{h+y}{d-x}-\tan ^{-1} \frac{h+y}{d+t-x}\right]
\end{aligned}
$$

One can add the field contribution by the three bars (Fig. 1) to get the total field as

$$
B_{z}(x, y)=A\left[\tan ^{-1} \frac{h_{3}-y}{d_{1}-x}-\tan ^{-1} \frac{h_{1}-y}{d_{1}-x}-\right.
$$




$$
\begin{aligned}
& \tan ^{-1} \frac{h_{3}-y}{d_{3}-x}+\tan ^{-1} \frac{h_{1}-y}{d_{3}-x}+\tan ^{-1} \frac{h_{3}+y}{d_{1}-x}- \\
& \tan ^{-1} \frac{h_{1}+y}{d_{1}-x}-\tan ^{-1} \frac{h_{3}+y}{d_{3}-x}+\tan ^{-1} \frac{h_{1}+y}{d_{3}-x}- \\
& \tan ^{-1} \frac{h_{2}-y}{d_{1}-x}-\tan ^{-1} \frac{h_{1}-y}{d_{1}-x}-\tan ^{-1} \frac{h_{2}-y}{d_{2}-x}+ \\
& \tan ^{-1} \frac{h_{1}-y}{d_{2}-x}-\tan ^{-1} \frac{h_{2}+y}{d_{1}-x}-\tan ^{-1} \frac{h_{1}+y}{d_{1}-x}- \\
& \tan ^{-1} \frac{h_{2}+y}{d_{2}-x}+\tan ^{-1} \frac{h_{1}+y}{d_{2}-x}+\tan ^{-1} \frac{h-y}{l_{1}+x}- \\
& \left.\tan ^{-1} \frac{h-y}{l_{2}+x}+\tan ^{-1} \frac{h+y}{l_{1}+x}-\tan ^{-1} \frac{h+y}{l_{2}+x}\right]
\end{aligned}
$$

$$
\begin{gathered}
B_{z}\left(x_{2}, 0\right)-B(0,0)-G x_{2}<\text { Tolerance } \\
\ldots \ldots \ldots \ldots \ldots \ldots \ldots \ldots \ldots \ldots \ldots \ldots \ldots \ldots \ldots \ldots \ldots \\
B_{z}\left(x_{1}, y_{m}\right)-B(0,0)-G x_{1}<\text { Tolerance }
\end{gathered}
$$

Since $\left[B_{z}(x, y)-B(0,0)-G x\right]$ can be either positive or negative, we have to use another set of constraints

$$
-B_{z}\left(x_{1}, 0\right)+B(0,0)+G x_{1}<\text { Tolerance }
$$

We want that the field due to the channel should have a specific value $B(0,0)$ at the chosen centre and have a chosen field gradient $G$, i.e.,

$$
B_{z}(x, y)=B(0,0)+G x
$$

for all $y$. The actual field of the channel will somewhat deviate from this and our endeavour is to minimize the deviation by optimizing the channel geometry.

\section{LINEAR PROGRAMMING METHOD}

The parameters which have been optimized are $h, h_{1}, h_{2}, h_{3}, d_{1}, d_{2}$ and $d_{3}$. As seen from eqn.( 3 ), $B_{z}(x, y)$ is not a linear function of these parmeters and so is not directly amenable to linear programming. So we first linearize the field in a small range. In the neighbourhood of any set of the parmeters we can expand the function $B_{z}(x, y)$ to get

$$
\begin{gathered}
B_{z}(x, y)=B_{z 0}(x, y)+\Delta h\left(\frac{\partial B_{z}}{\partial h}\right)_{0} \\
+\Delta h_{1}\left(\frac{\partial B_{z}}{\partial h_{1}}\right)_{0}+\Delta h_{2}\left(\frac{\partial B_{z}}{\partial h_{2}}\right)_{0}+\Delta h_{3}\left(\frac{\partial B_{z}}{\partial h_{3}}\right)_{0} \\
+\Delta d_{1}\left(\frac{\partial B_{z}}{\partial d_{1}}\right)_{0}+\Delta d_{2}\left(\frac{\partial B_{z}}{\partial d_{2}}\right)_{0}+\Delta d_{3}\left(\frac{\partial B_{z}}{\partial d_{3}}\right)_{0}
\end{gathered}
$$

where $B_{z 0}(x, y)$ is the field at $(x, y)$ with the initial set of parameters. This is linear in terms of the increments $\Delta h, \Delta h_{1}$ etc. and so one can now appply linear programming to minimize $\left|B_{z}(x, y)-B(0,0)-G x\right|$. We use the constraint that the deviations from the design field profile should be smaller than the tolerance for $-x_{m} \leq x \leq x_{m}$ where $2 x_{m}$ is the maximum beam width in the horizontal plane. The constraint is used at another parallel plane also with $y=y_{m}$ where $2 y_{m}$ is the vertical width of the beam. We have taken $x_{m}=0.3 \mathrm{~cm}$ and $y_{m}=0.2 \mathrm{~cm}$. This is done at a number of points, $x_{1}, x_{2}, \ldots$ within $-x_{m}$ to $x_{m}$ to get the set of constraints as

$$
B_{z}\left(x_{1}, 0\right)-B(0,0)-G x_{1}<\text { Tolerance }
$$

We have also put restrictions to the ranges within which the parameters are allowed to vary. The simplex technique is used for solving this problem of for minimizing Tolerance. The standard simplex technique maximizes the value of a variable. Since we want to minimize a variable, we do it by introducing a variable Tol given by

$$
\text { Tolerance }=\text { Tolmax }- \text { Tol }
$$

where Tolmax is an arbitrarily large constant. Tol can now be maximized for minimizing Tolerance. The simplex algorithm gives a set of corrections $\Delta h, \Delta h_{1}$ etc. We do the calculation in an iterative manner, in which a small fraction of the correction is applied at each step. It is necessary to specify whether one wants to increase or decrease any particular parameter. If in any trial a parameter remains unchanged, its sign is reversed in the next trial.

\section{RESULTS AND DISCUSSION}

We started with the geometry of the channel used in K500 machine at MSU[6] (Table 1). It has $B(0,0)=-1.214$ $\mathrm{kG}$ and $G(0,0)=3.3 \mathrm{kG} / \mathrm{cm}$. Fig. 2 shows the geometry of the optimized channel. The dotted geometry is that of the unoptimized one. We have not tried to change the parameters $l_{1}$ and $l_{2}$ of Bar 1 as these will change the field appreciably inside the cyclotron. However, the other six parmaters have little influence on the field on the left of Bar 1 and have been optimized.

Fig. 3(a) compares the deviation of the field from the design field profile. Fig. 3(b) shows that the deviation in gradient as a function of $x$. The deviation in the optimized channel comes down to $0.20 \mathrm{kG} / \mathrm{cm}$ from $0.83 \mathrm{kG} / \mathrm{cm}$ in the unoptimized channel. The superiority of the optimized channel is obvious. The optimization also decreases the values of the unwanted higher harmonic field components, responsible for the nonlinear effects. The optimized geometry gives a variation of $2.6 \mathrm{kG} / \mathrm{cm}^{2}$ in the sextupolar component compared to $9.1 \mathrm{kG} / \mathrm{cm}^{2}$ in the unoptimized geometry (Fig. 3(c)).

Fig. 4 compares the estimated beam ellipses when a beam of emittance of $9 \mathrm{~mm}$.mrad passes through the 9 optimized and unoptimized channels. The ellipse gets distorted appreciably by the unoptimized channels and the overall emiitance increases by about $30 \%$. 


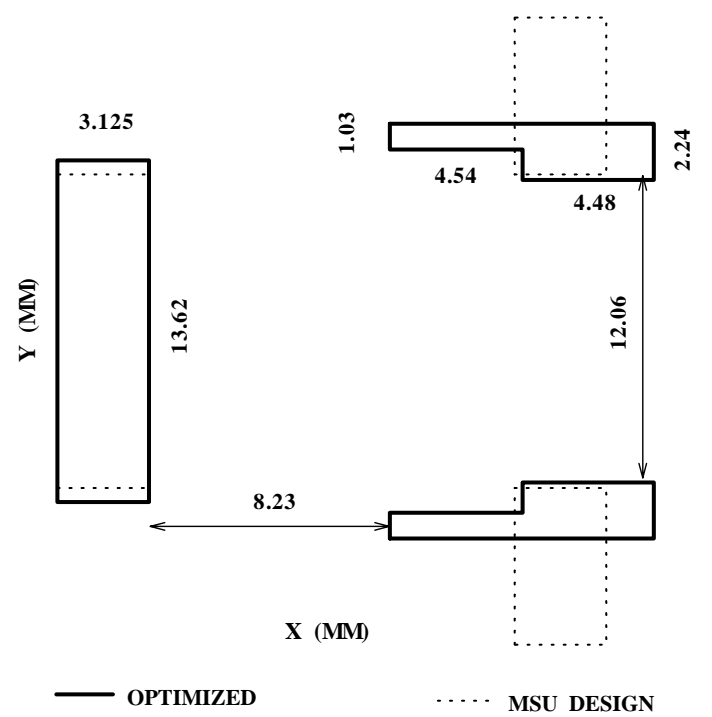

Figure 2: Optimized channel geometry compared with the MSU channel.

Table 1: Channel dimensions and field quality

\begin{tabular}{lll}
\hline \hline Parameter & Unoptimized & Optimized \\
\hline$h(\mathrm{~cm})$ & 0.625 & 0.681 \\
$h_{1}(\mathrm{~cm})$ & 0.625 & 0.603 \\
$h_{2}(\mathrm{~cm})$ & 0.625 & 0.724 \\
$h_{3}(\mathrm{~cm})$ & 1.25 & 0.827 \\
$d_{1}(\mathrm{~cm})$ & 0.625 & 0.198 \\
$d_{2}(\mathrm{~cm})$ & 0.625 & 0.652 \\
$d_{3}(\mathrm{~cm})$ & 0.9375 & 1.10 \\
$l_{1}(\mathrm{~cm})$ & 0.625 & 0.625 \\
$l_{2}(\mathrm{~cm})$ & 0.9375 & 0.9375 \\
\hline Max. $\Delta G(\mathrm{kG} / \mathrm{cm})$ & 0.840 & 0.206 \\
\hline \hline
\end{tabular}

The present method can be applied to other simple geometries also, e.g., in a channel which only lowers the field but does not produce any gradient or a channel of any required field profile.

\section{ACKNOWLEDGEMENT}

We thank Mr. M.K. Dey for his help in computing the beam ellipses.

\section{REFERENCES}

[1] R.K. Bhandari, Proceedings of the 15th. International Conference on Cyclotrons and their Applications, Caen, 1998, p.692.

[2] E. Fabrici, D. Johnson and F.G. Resmini, Nuclear Instruments and Methods /bf 184(1981)301.

[3] M. Odera, Y. Miyazawa, T. Tonuma, M. Hemmi and O. Terajima, Nuclear Instruments and Methods 65(1968)247.

[4] C.R. Hoffman, IEEE Trans. on Nuclear Science, NS24(1977)1470.

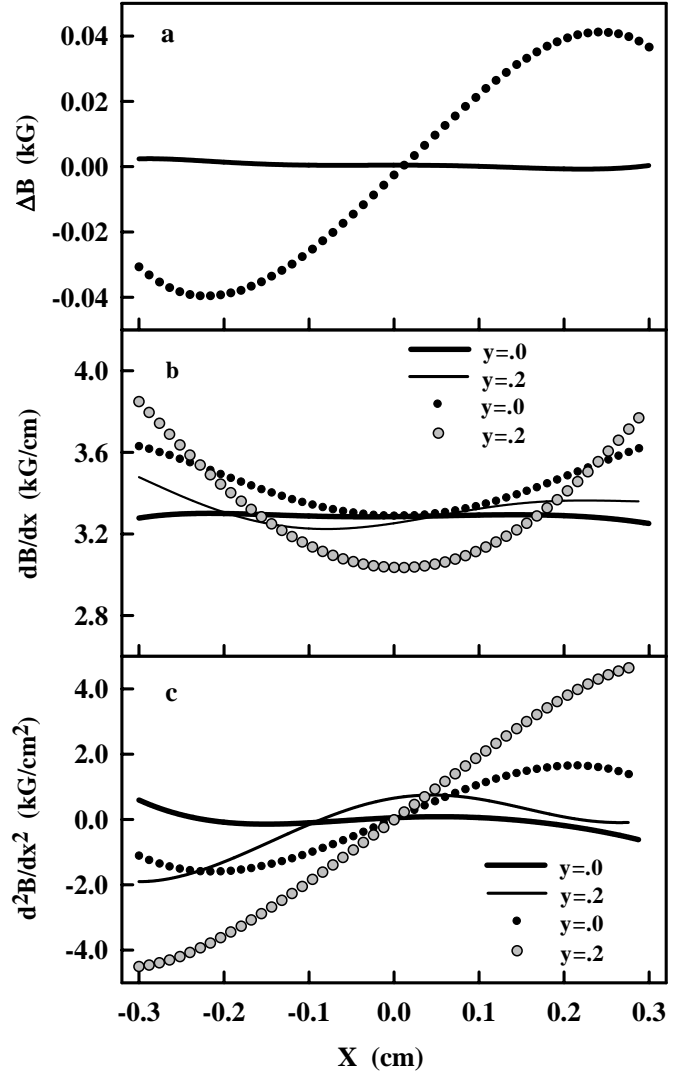

Figure 3: Comparison of the deviations in the (a) field, (b) field gradient, and (c) second gradient of the field. The solid curves are for the optimized channel and the dotted curves are for the unoptimized channel.

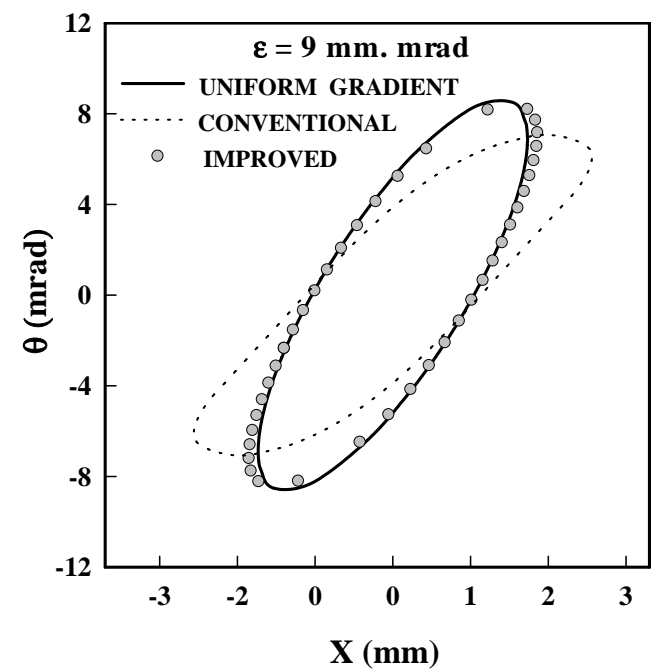

Figure 4: Comparison of beam ellipses as the exit of the 9 optimized and unoptimized channels.

[5] M.M. Gordon, D.A. Johnson and V. Taivassalo, MSU Cyclotron Laboratory Annual Report (1983-84), p.218.

[6] G. Bellomo, Proceedings of the 13th International Conference on Cyclotrons and their Applications, Vancouver, 1992, p.592.

[7] P.R. Sarma, V.S. Pandit and R.K. Bhandari, Nuclear Instruments and Methods 455(2000)539. 